\title{
Message Broadcast using Optical Orthogonal Codes in Vehicular Communication Systems
}

\author{
Farzad Farnoud, Behnam Hassanabadi, and Shahrokh Valaee \\ The Edward S. Rogers Sr. Department of Electrical and Computer Engineering \\ University of Toronto, 10 King's College Road, Toronto, ON, M5S 3G4, Canada \\ e-mail:\{frd,behnam,valaee\}@ comm.utoronto.ca
}

\begin{abstract}
Broadcast communication is considered to be especially important in delivering safety messages in vehicular environments. In this paper, we introduce and explore a new method for message broadcast based on repetition. The proposed method uses optical orthogonal codes in vehicular broadcast communications to increase the probability of detection and reduce reception delay. We formulate a general framework in which we can examine and evaluate the performance of broadcast schemes based on repetition. This framework is used to compare our method to other proposed broadcast methods.
\end{abstract}

Index Terms-Vehicular communications, optical orthogonal codes, random broadcast, safety messaging

\section{INTRODUCTION}

Vehicular communication networks are attracting increased attention because of their potential to provide safer and faster road travels. Governments and manufacturers, alike, increasingly invest in vehicular communications. This has become a motivation for researchers to design and evaluate networks for vehicular environments. One of the major characteristics of vehicular networks is the need to provide reliable service with low-latency for safety-related messages.

Safety messages are often considered to be broadcast messages intended for all nearby vehicles. The fact that location of vehicles determines the destination of messages can be exploited in the design of a network for vehicular communication. In fact, in many cases a single-hop broadcast messaging may be enough for the purpose of safety messages.

In general, broadcasting nodes, do not use RTS/CTS as a collision avoidance scheme. Also acknowledgements are not typically required for broadcast packets. This results in less reliable communication compared to unicast transmission. Reliability is especially very important in vehicular environments where the purpose of communication is to provide safety. Even if a transmitter decides to use RTS/CTS, it will not be efficient because many RTS/CTS messages will result in too much overhead, particularly if the broadcast messages are short, as in vehicular communication.

To increase reliability of broadcast messaging, retransmission of broadcast messages has been suggested [?]. Each packet is transmitted several times in a frame containing $L$ time slots. The duration of each time slot is equal to the transmission time of a packet. The transmission pattern in a frame can be represented by a code which is a sequence of zeros and ones with length $L$ and Hamming weight $w$, where

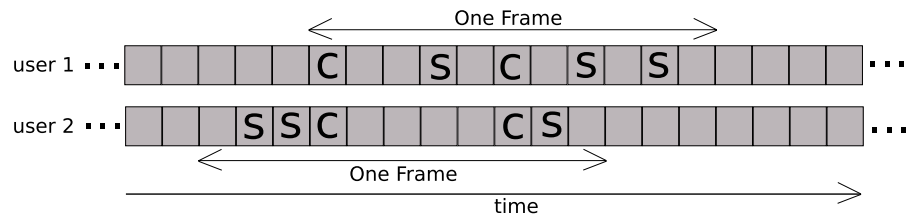

Fig. 1. Illustration of a broadcast scheme with $w=5$ and $L=11$; 'c' denotes a collision and 's' denotes a successful transmission.

$w$ is the total number of transmissions of a packet. Figure 1 shows two users transmitting simultaneously in a frameasynchronous setup. The code of user1 is 10010101010 and the code of user 2 is 01110000110 . In this figure, collision (when both users transmit simultaneously) are marked as ' $c$ ' and successes (single user transmitting) are marked as 's'. A packet is successfully transmitted if there is at least one slot marked as 's' over the transmission frame.

To avoid collisions, and hence performance degradation, we introduce a scheme that chooses the retransmission patterns intelligently with least possible correlation so as to minimize the probability of collision. Our approach is similar to code division multiple access systems; however instead of expanding every bit in time, packets are retransmitted with patterns which are almost orthogonal to each other. This is analogous to positive optical systems [2], where signals do not add to zero. Similarly, in a packet network, simultaneous transmissions result in collision. For this reason we use the same family of codes as used in optical code division multiple access, namely Optical Orthogonal Codes (OOC) [3]. This family of codes has been extensively studied and its good properties are well-known.

\section{General AnAlysis of Multiple BROADCAST SCHEMES}

In this section, we provide a general method for the analysis of multiple broadcast schemes with frame length $L$ and weight $w$. The desired user is susceptible to interference from other users in $w$ slots in which it transmits a packet. The interference pattern of user $i, u_{i}$, can be shown by a subset of $\{1,2, \cdots, w\}$, denoted by $I_{i}$. The elements of $I_{i}$ indicate the 
time slots that are identified as collision with the desired user, where the reference for numbering time slots is the desired user. For example, in the following code space, shown in a matrix form where each row corresponds to the code of a user,

$$
\left(\begin{array}{llllll}
\mathbf{1} & \mathbf{0} & \mathbf{1} & \mathbf{0} & \mathbf{1} & \mathbf{0} \\
0 & 0 & 1 & 1 & 1 & 0 \\
1 & 1 & 1 & 0 & 0 & 0 \\
0 & 1 & 1 & 0 & 0 & 1 \\
1 & 0 & 1 & 0 & 0 & 1
\end{array}\right)
$$

assuming $u_{1}$ (the first row) is the desired user, we have $I_{2}=$ $\{2,3\}, I_{3}=\{1,2\}, I_{4}=\{2\}$, and $I_{5}=\{1,2\}$.

Let $S_{r}$ be a subset of $\{1,2, \cdots, w\}$, where $1 \leq r \leq 2^{w}$. We decompose all other users (excluding the desired user, $u_{1}$ ) into $2^{w}$ disjoint sets, denoted by $\mathcal{A}_{r}$, where $\mathcal{A}_{r}=\left\{u_{i} \mid I_{i}=S_{r}\right\}$. That is $\mathcal{A}_{r}$ includes all users that interfere with the desired user with a pattern shown by the corresponding subset $S_{r}$. In the example above for $S_{r}=\{1,2\}$, we have $\mathcal{A}_{r}=\left\{u_{3}, u_{5}\right\}$. Note that for $r \neq r^{\prime}, \mathcal{A}_{r} \cap \mathcal{A}_{r^{\prime}}=\emptyset$. Also, $\Gamma_{S_{r}}$ is defined as the number of users that interfere with the desired user with pattern $S_{r}$. In other words, $\Gamma_{S_{r}}$ is the cardinality of $\mathcal{A}_{r}$. In example (1), $\Gamma_{\emptyset}=\Gamma_{\{1\}}=\Gamma_{\{3\}}=\Gamma_{\{1,3\}}=\Gamma_{\{1,2,3\}}=0$, $\Gamma_{\{2\}}=\Gamma_{\{2,3\}}=1$, and, $\Gamma_{\{1,2\}}=2$.

The probability of interference with the specific pattern $S_{r}$ is shown by $p_{S_{r}}$. Assuming some degree of randomness in the codes, $p_{S_{r}}$ only depends on the cardinality of $S_{r}$, shown by $\left\|S_{r}\right\|$. Hence $p_{S_{r}}=p_{S_{t}}$, as long as $\left\|S_{r}\right\|=\left\|S_{t}\right\|$. With small abuse of notation, we can write $p_{\left\|S_{r}\right\|}=p_{S_{r}}$.

Assuming different interfering users have independent interfering patterns,

$$
\operatorname{Pr}\left(\Gamma_{S_{r}}=0\right)=\left(1-p_{\left\|S_{r}\right\|}\right)^{N-1}
$$

where $N$ is the number of users in the system.

The number of interferes in the $i$ th slot (out of $w$ slots) is denoted by $Z_{i}$ which can be written as

$$
Z_{i}=\sum_{r=1}^{2^{w}} \Gamma_{S_{r}} I\left(\{i\} \cap S_{r} \neq \emptyset\right)
$$

For example,

$$
\begin{aligned}
Z_{1}=\Gamma_{\{1\}}+ & \Gamma_{\{1,2\}}+\Gamma_{\{1,3\}}+ \\
& \cdots+\Gamma_{\{1, w\}}+\Gamma_{\{1,2,3\}}+\cdots+\Gamma_{\{1, \cdots, w\}}
\end{aligned}
$$

In example (1), $Z_{1}=\Gamma_{\{1\}}+\Gamma_{\{1,2\}}+\Gamma_{\{1,3\}}+\Gamma_{\{1,2,3\}}=2$, $Z_{2}=\Gamma_{\{2\}}+\Gamma_{\{1,2\}}+\Gamma_{\{2,3\}}+\Gamma_{\{1,2,3\}}=4, Z_{3}=\Gamma_{\{3\}}+$ $\Gamma_{\{1,3\}}+\Gamma_{\{2,3\}}+\Gamma_{\{1,2,3\}}=1$.

Assuming $Z_{i}$ 's are statistically identical, probability of a successful transmission can be written as

$$
\begin{aligned}
P_{s}=\operatorname{Pr}\left(Z_{1}=0 \vee Z_{2}=0 \vee \cdots \vee Z_{w}=0\right)= \\
\sum_{k=1}^{w}(-1)^{k+1}\left(\begin{array}{c}
w \\
k
\end{array}\right) \operatorname{Pr}\left(Z_{1}=0, \cdots, Z_{k}=0\right)
\end{aligned}
$$

Using (3), we can write (6) where we have used the fact that all the patterns $S_{r}$ with same cardinality happen with equal probability. In (6), $I($.$) is the identifier function which is 1$ if the condition inside the brackets is true, and 0 otherwise, and $p_{j}$ is the probability of a set $S_{r}$ with $\left\|S_{r}\right\|=j$. We generalize (6) to write

$$
\begin{aligned}
\operatorname{Pr}\left(Z_{1}\right. & \left.=0, \cdots, Z_{k}=0\right) \\
& =\operatorname{Pr}\left(\sum_{r=1}^{2^{w}} \Gamma_{S_{r}} I\left(\{1, \cdots, k\} \cap S_{r} \neq \emptyset\right)=0\right) \\
& =\operatorname{Pr}\left(\bigwedge_{\{1, \cdots, k\} \cap S_{r} \neq \emptyset} \Gamma_{S_{r}}=0\right) \\
& =\left(1-\sum_{j=1}^{w} p_{j} \sum_{i=1}^{\min (j, k)}\left(\begin{array}{c}
k \\
i
\end{array}\right)\left(\begin{array}{c}
w-k \\
j-i
\end{array}\right)\right)^{N-1}
\end{aligned}
$$

Finally, by substituting (7) in (5), $P_{s}$ is written as

$$
\begin{aligned}
P_{s}= & \sum_{k=1}^{w}(-1)^{k+1}\left(\begin{array}{c}
w \\
k
\end{array}\right) \\
& \left(1-\sum_{j=1}^{w} p_{j} \sum_{i=1}^{\min (j, k)}\left(\begin{array}{c}
k \\
i
\end{array}\right)\left(\begin{array}{c}
w-k \\
j-i
\end{array}\right)\right)^{N-1}
\end{aligned}
$$

The above formula is general and is valid for broadcast communications using packet repetitions. In the following sections, we use (8) to evaluate the performance of our scheme and compare it to the method proposed in [4].

\section{BROADCAST METHODS}

\section{A. Broadcast using OOC's}

Central to the idea of OOC's is designing positive codes with minimum auto- and cross-correlation. Consider a family of OOC codes with length $L$ and weight $w$. Auto-correlation and cross correlation between two OOC codes, $u$ and $v$, are bounded by $\lambda_{a}$ and $\lambda_{c}$, respectively, i.e. for any code $u$

$$
\max _{k} \sum_{j=1}^{L} u_{j} \cdot u_{j \oplus k} \leq \lambda_{a}
$$

and for $u \neq v$

$$
\max _{k} \sum_{j=1}^{L} u_{j} \cdot v_{j \oplus k} \leq \lambda_{c}
$$

where $\oplus$ is addition modulo $L$. Imposing (9) and (10) on all possible codes will result in eliminating some codes and hence reducing the number of available codes. For $\lambda=\lambda_{a}=\lambda_{c}$ the number of codes is upper bounded by [1]

$$
N \leq \frac{(L-1)(L-2) \cdots(L-\lambda)}{w(w-1)(w-2) \cdots(w-\lambda)} .
$$

Here, we consider only the case with $\lambda=2$.

To evaluate the performance of broadcast using OOC's, we must find $p_{i}$ and substitute in (8). Note that the $p_{i}$ corresponds only to one interfering user. Assume the intended user starts transmission at time $t_{0}$. Packets transmitted by the interfering user in $\mathcal{T}=\left[t_{0}-T_{f}, t_{0}+T_{f}\right)$ may interfere with a slot in which the intended user is transmitting. Considering Poisson traffic model with mean $\mu_{p}$ and assuming $\left(2 \mu_{p} T_{f}\right)^{2} \ll 1$, the interfering user generates one packet in $\mathcal{T}$ with probability 


$$
\begin{aligned}
\operatorname{Pr}\left(Z_{1}=0\right) & =\operatorname{Pr}\left(\sum_{r=1}^{2^{w}} \Gamma_{S_{r}} I\left(\{1\} \subseteq S_{r}\right)=0\right) \\
& =\operatorname{Pr}\left(\Gamma_{\{1\}}=0, \Gamma_{\{1,2\}}=0, \Gamma_{\{1,3\}}=0, \cdots, \Gamma_{\{1, w\}}=0, \Gamma_{\{1,2,3\}}=, \cdots, \Gamma_{\{1, \cdots, w\}=0}\right) \\
& =\left(1-\left[p_{1}\left(\begin{array}{c}
w-1 \\
0
\end{array}\right)+p_{2}\left(\begin{array}{c}
w-1 \\
1
\end{array}\right)+p_{3}\left(\begin{array}{c}
w-1 \\
2
\end{array}\right)+\cdots+p_{w}\left(\begin{array}{c}
w-1 \\
w-1
\end{array}\right)\right]\right)^{N-1}
\end{aligned}
$$

$1-e^{-2 \mu_{p} T_{f}}$. Alternatively, we can assume packets generated when a packet is being transmitted are discarded.

Assume another user is interfering with the desired user, and let $\mathbf{D}$ be the number of slots that the frames of the two users overlap and $\ell$ be the number of repetitions that the intended user transmits during the overlap. For example in Figure 1, assuming user 1 is the desired user, $\mathbf{D}=8$ and $\ell=4$. The probability mass function of $\mathbf{D}$ can be written as:

$$
\operatorname{Pr}(\mathbf{D}=d)= \begin{cases}\left(1-e^{-2 \mu_{p} T_{f}}\right) \frac{2}{2 L-1} & 1 \leq d<L \\ \left(1-e^{-2 \mu_{p} T_{f}}\right) \frac{1}{2 L-1} & d=L \\ e^{-2 \mu_{p} T_{f}} & \text { otherwise }\end{cases}
$$

Also:

$$
\operatorname{Pr}(\ell=l \mid \mathbf{D}=d)=\frac{\left(\begin{array}{l}
d \\
l
\end{array}\right)\left(\begin{array}{l}
L-d \\
w-l
\end{array}\right)}{\left(\begin{array}{l}
L \\
w
\end{array}\right)}
$$

and

$$
\operatorname{Pr}(\mathbf{I}=i \mid \ell=l)=\frac{\sum_{j=0}^{2-i}\left(\begin{array}{c}
l \\
i
\end{array}\right)\left(\begin{array}{c}
w-l \\
j
\end{array}\right)\left(\begin{array}{c}
L-w \\
w-i-j
\end{array}\right)}{\left(\begin{array}{c}
w \\
0
\end{array}\right)\left(\begin{array}{c}
L-w \\
w
\end{array}\right)+\left(\begin{array}{c}
w \\
1
\end{array}\right)\left(\begin{array}{c}
L-w \\
w-1
\end{array}\right)+\left(\begin{array}{c}
w \\
2
\end{array}\right)\left(\begin{array}{c}
L-w \\
w-2
\end{array}\right)}
$$

where $\mathbf{I}$ is the number of collisions caused by the interfering user. $^{1}$

Using the total probability theorem, the values for $p_{i}$ can be calculated by substituting (12), (13), and (14) into the following formula

$$
\begin{aligned}
& \operatorname{Pr}(\mathbf{I}=i)=\sum_{l} \operatorname{Pr}(\mathbf{I}=i \mid \ell=l) \operatorname{Pr}(\ell=l) \\
= & \sum_{l}\left(\operatorname{Pr}(\mathbf{I}=i \mid \ell=l) \sum_{d} \operatorname{Pr}(\ell=l \mid \mathbf{D}=d) \operatorname{Pr}(\mathbf{D}=d)\right)
\end{aligned}
$$

Note that $p_{i}$ can be found as

$$
p_{i}=\frac{\operatorname{Pr}(\mathbf{I}=i)}{\left(\begin{array}{c}
w \\
i
\end{array}\right)}
$$

\section{B. Broadcast with random codes}

In this method, $w$ slots for transmission are chosen randomly among $L$ slots [4]. The analysis for this method is similar to that of OOC's except that (14) must be substituted by

$$
\operatorname{Pr}(\mathbf{I}=i \mid \ell=l)=\frac{\left(\begin{array}{l}
l \\
i
\end{array}\right)\left(\begin{array}{l}
L-l \\
w-i
\end{array}\right)}{\left(\begin{array}{l}
L \\
w
\end{array}\right)}
$$

${ }^{1}\left(\begin{array}{l}a \\ b\end{array}\right)$ is zero if $b>a$.

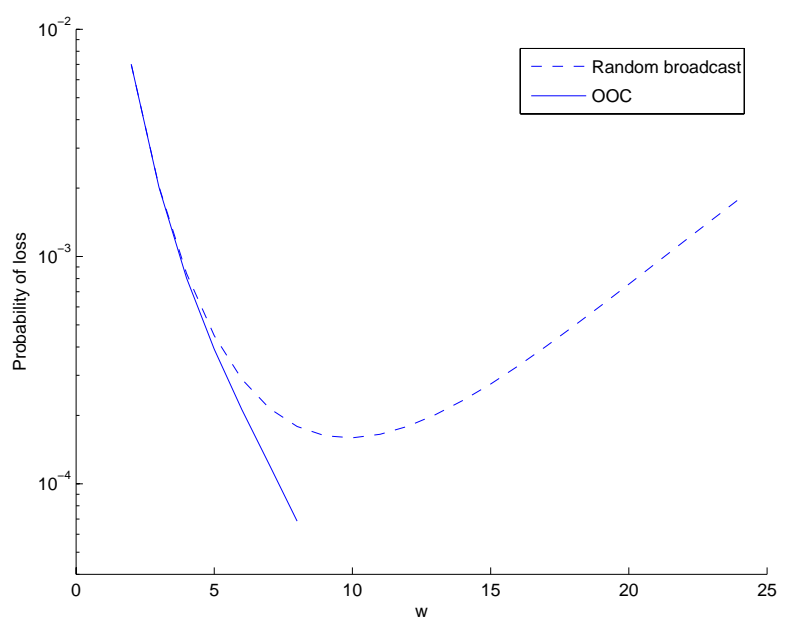

Fig. 2. The probability of loss vs. $w$

Therefore $p_{i}$ is found by substituting (12), (13), and (17) into (15) and then using (16).

\section{Performance eVAluation AND Numerical RESULTS}

Substituting $p_{i}$ for Random broadcast and OOC into (8) gives the probability of successful transmission. For given $L$,

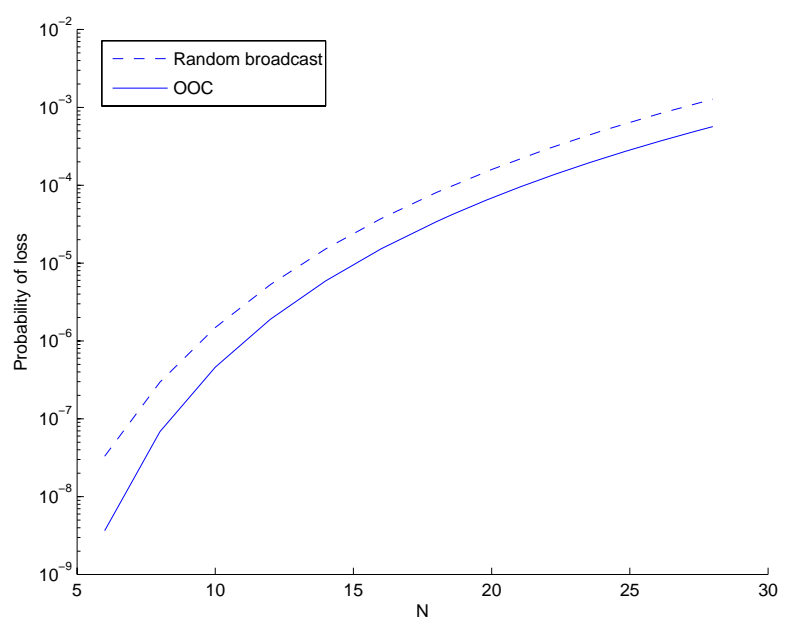

Fig. 3. The probability of loss vs. $N$ 
$N$, and $\mu_{p}$, for each scheme, we can find the optimum value of $w$ and compare the optimum performances.

For $L=100, N=20$, and $\mu_{p}=0.3 / T_{f}$, the packet loss probability from (8) is plotted for both cases in Figure IV. Note that for OOC, when $N$ is given, $w$ is limited by (11). It is observed that OOC can reach better performance with smaller $w$. The optimum for OOC occurs at $w=8$ and for random broadcast occurs at $w=10$. Therefore random broadcast keeps the channel busier by sending more packets and still has inferior performance compared to OOC.

In Figure IV with $L=100$, and $\mu_{p}=0.3 / T_{f}$, the two schemes are compared when the number of users is ranged from 6 to 20. Again it is seen that OOC provides better performance and less loss.

\section{REFERENCES}

[1] FRK Chung, JA Salehi, VK Wei, B.C. Res, and NJ Morristown. Optical orthogonal codes: design, analysis and applications. Information Theory, IEEE Transactions on, 35(3):595-604, 1989.

[2] K.P. Jackson, SA Newton, B. Moslehi, M. Tur, C.C. Cutler, JW Goodman, and HJ Shaw. Optical fiber delay-line signal processing. IEEE Transactions on Microwave Theory and Techniques, 33(3):193-210, 1985.

[3] JA Salehi, B.C. Res, and NJ Morristown. Code division multipleaccess techniques in optical fiber networks. I. Fundamental principles. Communications, IEEE Transactions on, 37(8):824-833, 1989.

[4] Qing Xu, Tony Mak, Jeff Ko, and Raja Sengupta. "medium access control protocol design for vehiclevehicle safety messages". Vehicular Technology, IEEE Transactions on, 56(2):499-518, 2007. 